\title{
La soberanía y los 4 jinetes del Apocalipsis
}

\author{
Ana Esther Ceceña \\ Universidad Nacional Autónoma de México \\ anacecena@gmail.com
}

\section{Resumen}

El diseño organizativo político-territorial del sistema-mundo capitalista forjó una configuración disciplinaria-cohesionadora en la que el Estado-nación se erigió como estructura central. Con el tiempo esta figura y la institucionalidad misma del capitalismo han sido cuestionadas o corroídas por los mismos agentes y fuerzas que las crearon o que no alcanzaron a ser disciplinados. En este texto se destacan los desafíos a la soberanía del Estado que emergen de la acción de cuatro sujetos: los supraestados (teoría del imperialismo), las corporaciones, el crimen organizado (los cárteles) y las fuerzas y societalidades subordinadas y en resistencia (teoría del colonialismo interno).

\section{Palabras clave}

1| soberanía 2| Estado 3| territorialidades en disputa 4| colonialismo

\section{Cita sugerida}

Ceceña, Ana Esther (2021). La soberanía y los 4 jinetes del Apocalipsis. Tramas y Redes, (1), 21-34, 101a. DOI: 10.54871/cl4c101a. 


\title{
A soberania e os 4 cavaleiros do Apocalipse
}

\section{Resumo}

O desenho organizacional politico-territorial do sistema mundial capitalista forjou uma configuração disciplinar coesa na qual o Estado-nação emergiu como a estrutura central. Com o tempo, essa figura e a própria institucionalidade do capitalismo foram questionadas ou corroídas pelos mesmos agentes e forças que as criaram ou que não foram disciplinados. Este texto destaca os desafios à soberania do Estado que emergem da ação de quatro sujeitos: os supra-estados (teoria do imperialismo), corporações, crime organizado (cartéis) e forças e sociedades subordinadas e na resistência (teoria do colonialismo interno).

\section{Palavras chave}

1| soberania 2| Estado 3| territorialidades em disputa $4 \mid$ colonialismo

\section{Sovereignty and the 4 Horsemen of the Apocalypse}

\begin{abstract}
The organization of the political and territorial design of the capitalistic world-system was forged by providing cohesion through discipline. In that structure the state-nation plays a central role. Over time, however, this state-nation and the institution of capitalism itself have been questioned and corroded by the very agents that created it, as well as by those that had not been fully disciplined. This article will highlight the challenges to state sovereignty that emerge from the action of four subjects: super-states (imperialism theory), corporations, organized crime (drug cartels) and subordinated societalities in resistance (inner colonialism theory).
\end{abstract}

\section{Keywords}

$1 \mid$ sovereignty $2 \mid$ state $3 \mid$ struggling territorialities $4 \mid$ colonialism 
Hey you, out there in the cold Getting lonely, getting old

Can you feel me?

Pink Floyd

\section{La ambigüedad de la soberanía}

Desde la primera globalización, que llevó a la universalización del Estadonación como forma de organización de las sociedades, la noción de soberanía acompaña los procesos estructurantes y atraviesa los conflictos que se producen en su devenir, sea por el afán de articular lo que es diverso -y muchas veces contradictorio- dentro de una unidad política y territorial delimitada y subordinada, sea por la arbitrariedad en la fijación de límites que un proceso de este carácter supone y la consecuente tendencia a la expansión de los núcleos de mayor fortaleza. No obstante, a pesar de que sigue siendo una noción imprecisa, con contenidos cambiantes a partir de la disposición política de quien la evoca, en términos generales el entendimiento contemporáneo de la soberanía la refiere al Estado y al territorio.

La soberanía de una nación o la soberanía de un pueblo, que son las dos formulaciones de mayor uso, suponen una identidad colectiva de difícil comprobación, atendiendo a la diversidad de culturas, etnias, historias particulares y pareceres de los grupos que la conforman, pero que funciona como elemento cohesionador en la defensa del territorio que se comparte o de intereses que en el juego internacional aparecen como representativos de la colectividad nacional en su conjunto. Así, los elementos que se reconocen como patrimonio nacional son sustento material de la soberanía de un pueblo o nación, y cualquier violación de fronteras territoriales, políticas, económicas, culturales u otras, de acuerdo con los límites demarcados por la identidad comunitaria y jurídica correspondientes, es considerada un atentado a la soberanía.

En este contexto, me interesa aportar algunas reflexiones sobre las amenazas a la soberanía de los países de América Latina y el Caribe, señalando a esta región geográfica como lugar de enunciación y abordando el análisis desde la disputa y reconfiguración de su dimensión territorial.

\section{Estados imperiales}

El capitalismo ha construido un orden mundial que homogeneiza criterios, impone reglas y formas de comportamiento a través de un intrincado 
sistema institucional que aparece como consensual pero que está atravesado por relaciones de poder, asimetrías y heterogeneidades que tienden a modificar los límites territoriales de las naciones soberanas y sus territorialidades o modos específicos de organización social en el territorio de acuerdo con sus modos de vida e idiosincrasia propios.

Los Estados-nación fueron, a la vez, un modo general de estructuración y disciplinamiento forjado en la constitución del sistema-mundo moderno capitalista, necesarios para la articulación y ordenamiento de intereses y dinámicas, $\mathrm{y}$ una modalidad de defensa de territorios particulares ante la voracidad de los poderes ajenos constituidos. Justo esto los hace aparecer como espacios de resistencia y de reivindicación de autodeterminación, supuestamente protegidos por el derecho internacional.

Es en este lugar metodológico donde se asienta la teoría del imperialismo que destacan las relaciones de fuerza entre los diferentes Estados que han configurado fronteras desde los inicios de este proceso, como modos de acotar conflictos, pero que también las subvierten permanentemente en la medida en que estas se convierten en obstáculos.

El imperialismo tiene una variedad de modalidades de intervención cuyo extremo es la disolución/anexión de territorios en principio ajenos. En una amplia gama que abarca todas las dimensiones de articulación institucional, tales modalidades se mueven desde las intervenciones bélicas, que se han sofisticado al grado de mostrar también un abanico de variantes, hasta intervenciones menos visibles y no consideradas como componentes de la guerra (convencional), aunque son cada vez más presentes y activas, como pueden ser, entre otras, los bloqueos comerciales o financieros. Todo esto junto es lo que en trabajos anteriores he denominado la guerra de espectro completo, retomando la formulación del Joint Chiefs of the Staff (Comando Conjunto) de Estados Unidos que usa el concepto de dominación de espectro completo aludiendo a la intervención concertada de todas las fuerzas militares. En mi concepción, lo militar se extiende hacia todas las esferas de definición de la vida en sociedad y subordina formas no militares a una lógica estratégica articulada. La estrategia de dominación se despliega tanto en la subordinación de la cultura y de la realidad alimenticia y agrícola, como en el desmonte de sentidos comunes configurados en la historia y la tradición de los pueblos para imponer un sentido homogeneizado y producido como mercancía global; asimismo en las decisiones de política económica, financiera, sanitaria o educativa, constituyendo todos estos los terrenos que militarmente se reconocen como el agua que hay quitarle al pez. Si alguna vez lo fue, la guerra ha dejado de ser solamente bélica. Incluso, considerando los grandes logros tecnológicos que otorgan un peso estratégico a los entramados del ciberespacio, las intervenciones tienen como terreno de mayor y más decisivo impacto la penetración de sistemas informáticos 
del identificado como enemigo. Esto puede referirse a sistemas de control de plantas nucleares, como ocurrió en Irán, o de sistemas financieros, de suministro de energía u otros, relevantes para poner en entredicho la capacidad soberana de una nación o para provocar una insurrección popular en contra del Estado. Las guerras se juegan, se ganan o se pierden en estos otros terrenos, como pusiera en evidencia traumática Vietnam y estudiara en tiempos antiguos Sun Tzu.

Los territorios se intervienen desde esta amplia gama de vertientes, buscando reconfigurar el sentido y la identidad colectivos, socavando, consecuentemente, la soberanía. Fuerza, cultura y control de los bienes básicos que aseguran la existencia son los tres componentes de una buena estrategia de rediseño y transformación en los criterios y modos de entendimiento y uso de los territorios.

Esta disputa sobre los territorios, que puede ser entendida como una confrontación de soberanías, es una constante en la dinámica del sistema-mundo moderno capitalista regida por un modo expansivo, apropiador/ concentrador y objetivador, con relaciones a la vez excluyentes y jerárquicas.

En esta tendencia a la ampliación, sea de la intervención directa, sea del control sobre los territorios, encontramos cuatro razones principales, que pueden variar su importancia relativa según las circunstancias específicas:

1. La apropiación de las riquezas asentadas en esos territorios, que dentro de un espectro amplio pueden ser lo que se considera como naturales, ya sean inorgánicas (minerales) u orgánicas (biodiversidad), pero también sociales, incluyendo en ello la diversidad de culturas y las capacidades y disposiciones para el trabajo. Cada momento histórico y tecnológico marca prioridades en la clasificación de estos bienes con respecto a su clasificación en la lista de estratégicos, básicos o prescindibles o sustituibles. De ahí también la importancia relativa de los distintos territorios.

2. La situación geográfica del territorio en cuestión, ya sea desde la perspectiva de las comunicaciones como en los casos de Suez, Panamá y posiblemente Tehuantepec, o territorios por donde pasan oleoductos o las rutas de suministro (las viejas y nuevas rutas de la seda) como Siria o Georgia. También se consideran aquí territorios como Afganistán, que es el vórtice entre las zonas petroleras y las de alimentación de coaliciones que amenazan la hegemonía ya establecida (vinculación entre Irak-Irán y China), como las rutas de suministro de la economía china o asiática en general. 
3. Ocupación estratégica de territorios que permitan romper la posibilidad de crear coaliciones competitivas con el poder hegemónico. Esta fue la característica de las guerras de Afganistán e Irak; la primera para ocupar un espacio en Asia Central después de la implosión de la Unión Soviética e impedir una extensión de China o un acercamiento entre China y la Federación Rusa; la segunda para impedir la formación de un poder árabe cohesionado sobre tierras de enorme riqueza natural y geoestratégica.

4. Territorios en los que emergen fuerzas antisistémicas con capacidad de organizar la vida colectiva en torno a principios de cohesión disidentes del capitalista, en confrontación o desapego que genera grietas en el orden mundial hegemónico. En este caso puede ubicarse a Venezuela, Cuba y Bolivia, pero también regiones (no necesariamente países) que alberguen grupos sociales amenazantes, donde sea necesario desmontar derivas bifurcatorias.

\section{Corporaciones}

Una segunda amenaza a las soberanías estatales está configurada por la actuación de las corporaciones. Es decir, se produce cuando el capital amenaza a las estructuras e instituciones creadas por él mismo. En estos casos, el sujeto se libera de las mediaciones societales para presentarse tal cual es: un sujeto voraz y expansivo, con agencia propia y en permanente transformación y empoderamiento.

Más allá de los límites y regulaciones estatales, las corporaciones van ocupando los espacios. Intervienen en todos los terrenos de construcción de la vida y modifican, así, los modos de pensar y las prácticas cotidianas. Regulan y transforman los hábitos alimenticios y el contenido de los alimentos, las movilidades, las interfaces de la comunicación y las relaciones sociales, los modos de concebir la salud, la enfermedad y la gestión de la buena vida, y también los modos de concebir/construir el espacio y el territorio. Van así subvirtiendo, moldeando y creando la territorialidad capitalista, recogiendo en ella no solo las homogeneidades, sino las disparidades, para combinarlas y ponerlas en juego en una totalidad articulada y complementaria. Son las corporaciones, a través del control no solo de la producción, sino también del consumo, las que van moldeando los sentidos de realidad, muchas veces contradiciendo o sobreponiéndose a las políticas del Estado (de cualquiera de los Estados).

Considerando que una de las más fuertes instituciones del capitalismo es la propiedad privada, asegurada y protegida por el Estado, pero 
reivindicada y ejercida por sus beneficiarios directos, fenómeno que se torna muy visible con las corporaciones de carácter agrícola o extractivo, los territorios de la Nación han ido pasando a manos privadas, quedando fuera de la jurisdicción del Estado. El caso de las minas o, en general, de la explotación del subsuelo, es el más elocuente: en muchas ocasiones lo que se otorga son concesiones de explotación por cincuenta años, renovables por otros cincuenta, que relegan los derechos del Estado que, de esta forma, durante un siglo no tiene la posibilidad de reclamar autoridad sobre los territorios concesionados y, cuando ya la tiene, lo que recupera son territorios vaciados.

No es nuevo que las empresas tengan propiedades o concesiones de partes del territorio tanto en sus países de origen como en el extranjero. No obstante, la escasez relativa de yacimientos o bienes naturales considerados recursos por su uso rentable, las dimensiones de su aprovechamiento y la consecuente competencia por su disposición excluyente, así como las condiciones tecnológicas que hicieron posible la globalización de los sistemas productivos, agudizaron la carrera por la ocupación privada del territorio.

La vanguardia en este proceso la tienen las corporaciones gigantes, con sistemas de producción globales y con capacidad y condiciones para modificar políticas públicas en los países donde van implantando sus huellas y para dictar políticas globales desde los organismos internacionales de regulación del orden mundial. Los mecanismos de intervención que tienen corporaciones que duplican, triplican, o más, el ingreso anual de muchos de los países en los que invierten son múltiples. Las amenazas de retirar inversiones, la presión de la competencia sobre empresas locales que generan oligopolios o monopolios y la creación de amplias redes de corrupción son algunos de los mecanismos recurrentes que utilizan estas poderosas corporaciones, además de que en muchos de los casos hay una actuación conjunta entre ellas y sus Estados sede. Esto llega incluso a ser motivo de estallido de guerras declaradas, como la de Irak, para el apoderamiento de sus yacimientos petroleros, entre otros.

Desde una perspectiva territorial, el impacto es mucho más profundo puesto que partes del territorio van quedando fuera de la jurisdicción del Estado nacional. En ocasiones las diversas legislaciones nacionales, como la laboral, ambiental u otras del mismo carácter, siguen teniendo vigencia sobre esos territorios, aunque en situación de relativa disputa. Es decir, visto desde el Estado-nación, su soporte territorial está siendo invadido o conculcado, lo que muestra una pérdida que puede metaforizarse con la imagen de un queso gruyer. Se entregan, voluntariamente o bajo presión, los territorios de densidad estratégica dentro de un país, de manera que sus riquezas o, lo que es lo mismo, el patrimonio de la nación sobre el que se construyen sus condiciones de supervivencia, fuerza y cohesión internas, se empobrece correlativamente. 
Esto pasa igualmente con la cesión de territorio para la instalación de bases militares extranjeras con el agravante de que, en estos casos, los territorios quedan estrictamente bajo la jurisdicción del ocupante, sin posibilidad siquiera de supervisión por parte del Estado anfitrión. Actualmente, recogiendo los datos de las bases militares oficiales y públicas de Estados Unidos que, por supuesto, no son todas, su extensión territorial alcanza 108,791 km2, el equivalente a la superficie de Guatemala $(108,889 \mathrm{~km} 2)$.

Las corporaciones gigantes deben ser entendidas como subsistemas integrados con reglas propias y, según las circunstancias, con escuelas, cines y supermercados dentro de sus instalaciones y con guardias blancas o ejércitos privados que las resguardan frente a las amenazas del exterior y contribuyen a imponer el orden interno, justificado por su desconfianza sobre la capacidad protectora que puede brindarles el Estado en las regiones sede. Es decir, tienen una especie de societalidad interna propia que se ejerce de acuerdo con las condiciones de cada lugar donde se asientan. Se pueden encontrar muchos ejemplos de esto en las minas peruanas o en las de diferentes lugares de África. En África en particular, los ejércitos privados funcionan con más intensidad ante la fragilidad de los Estados y la proliferación de bandas armadas. La resistencia de las poblaciones frente a este tipo de inversiones, que no solamente ocupan territorios sino que, simultáneamente, violentan las territorialidades previamente forjadas en ellos, es combatida, en general, lo mismo por los Estados nacionales que por las fuerzas privadas de la empresa.

Si se observa este proceso desde el mirador de la corporación se constata un traslape de territorialidades y societalidades que impone y diluye fronteras simultáneamente. La estructura de naciones, como señala el neoliberalismo al invocar la figura del mercado, se ha convertido en una camisa de fuerza institucional que contiene la expansión del capital, sin embargo, sigue siendo efectiva para el control de poblaciones mientras que el capital encuentra diversos mecanismos y rutas para traspasarla ya que las fronteras territoriales, culturales y políticas de las naciones no alcanzan a tener la fuerza que requerirían para regular a las corporaciones. Uno de los elementos más destacados de corrosión de la soberanía de los Estados, reforzado por instancias de regulación global, es el Centro Internacional de Arreglo de diferencias relativas a Inversiones (CIADI) del Banco Mundial, en el que en más del 90\% de los diferendos pierden la pelea los Estados, a quienes se les imponen multas altísimas, que a veces exceden el monto anual del Producto Interno Bruto (PIB).

Los territorios estáticos y regularmente continuos de los Estados se enfrentan a un sujeto de gran capacidad que ha construido territorios globales pero discontinuos. Yo los llamo territorios archipiélago por su conformación y características, por ser una diversidad integrada con forma 
más bien reticular, y que crece incorporando los pedazos de territorio que revisten densidad estratégica (Ceceña, 2017). O sea, son estructuraciones territoriales de altísimo valor, organizadas a la vez como regímenes privados de gobierno profundamente autoritarios y jerárquicos y que, vistos en las delimitaciones nacionales, serían una parte importante de los hoyos del queso gruyer: el no-Estado (o el otro Estado) dentro del territorio del Estado-nación.

Los territorios archipiélago corporativos son instancias de poder de tan alto nivel que apenas se equiparan con los Estados más poderosos del mundo con los que mantienen relaciones rara vez de subordinación, muchas veces colaborativas $y$, por tanto, tienen una agencia privilegiada (ver el caso de la Exxon Mobil como ejemplo paradigmático). Con respecto a los otros Estados su relación es desde una posición de fuerza y con capacidad para imponer o modificar políticas públicas y hasta límites territoriales (ver los casos del Esequibo, entre Venezuela y Guyana con nada menos que la Exxon en el centro; la partición de Sudán y otros similares).

La capacidad económica y, con ello, la agencia política de estas corporaciones gigantes queda de manifiesto al comparar sus ingresos con los de los Estados. Las veinte mayores corporaciones, en 2019, ${ }^{1}$ tienen un ingreso por ventas de 5.818.797 millones de dólares, lo que las coloca solo debajo del PIB de Estados Unidos (21.433.225 mdd) y China (14.279.937 mdd), de acuerdo con las mediciones de la revista Fortune (2019) y del Banco Mundial (2019). La región de América Latina y el Caribe completa alcanza un monto de 5.786 .727 millones de dólares de PIB generado en 2019, y sus negociaciones con el mundo están individualizadas, de manera que, incluso países como Brasil, con un PIB de 1.877.811 mdd, se encuentran en una situación asimétrica desfavorable frente a las corporaciones.

\section{Crimen organizado}

Un tercer sujeto destacado en el mundo contemporáneo es el genéricamente denominado crimen organizado, cuya expresión estructural son los cárteles. Como manifestación extrema de la mercantilización, el crimen organizado trafica con drogas, armas, personas y cuerpos, asumiendo actividades y negocios de gran rendimiento pero proscritos desde el punto de vista de

\footnotetext{
${ }^{1}$ Las primeras veinte corporaciones de la lista de Fortune en 2019, que es el dato con el que trabajamos, son Walmart, Sinopec Group, State Grid, China National Petroleum, Royal Dutch Shell, Saudi Aramco, Volkswagen, British Petroleum, Amazon, Toyota Motor, ExxonMobil, Apple, CVS Health, Berkshire Hathaway, UnitedHealth Group, McKesson, Glencore, China State Construction Engineering, Samsung Electronics y Daimler.
} 
la legalidad instituida. ${ }^{2}$ Esto lo hace, ya de entrada, un sujeto que de origen es un otro con respecto a los poderes institucionales a pesar de que, en los hechos, ha sido auspiciado y protegido por el Estado que a la vez lo condena, y con el que mantiene una imbricación colaborativa.

El tipo de mercancías con las que trafica este sujeto o la calidad especial de sus insumos, que en algunos casos son personas (llevando hasta el límite último las reflexiones de Marx sobre las mercancías ficticias), lo coloca en una situación de abuso extremo que no puede ser tolerada o justificada por las instituciones pero que, más allá de la peculiaridad de su materia de trabajo, no se diferencia demasiado de las otras modalidades de acumulación de capital.

No obstante, aun considerándolo una especie de excrecencia con respecto a la maquinaria general del capitalismo, su presencia no es circunstancial. Una paulatina instalación del uso de drogas y armas en los sentidos comunes y en las prácticas sociales anuncia su reconocimiento como parte de la regularidad de la vida. Así también ocurre con las diversas modalidades de trabajo forzado y el uso de los cuerpos, más aun después de los estragos sociales provocados por la pandemia o encubiertos en ella.

Es de suyo conocido cómo los dineros del crimen organizado determinan la política y someten a los Estados, designan autoridades civiles, subordinan fuerzas armadas y cuerpos de seguridad e imponen nuevas geografías. Rutas, aeropuertos e infraestructura pasan a ser controlados por estos traficantes bajo la protección de las fuerzas militares, tanto como decisiones políticas y prácticas y normativas sociales.

Las llamadas fuerzas del crimen organizado operan en amplios territorios que han ido transformando. Ellas establecen las reglas internas, a contrapelo de las que rigen en el ámbito nacional. Instauran políticas sociales a su sazón igualmente que sanciones, castigos y disciplinamientos forzosos como la cesión de ciertas tierras, la venta de sus productos, actividades de información y vigilancia o hasta el consentimiento para matrimonios no necesariamente deseados por alguna de las partes. Es decir, imponen una societalidad propia dentro del Estado, sin descuidar el hecho de que cuentan con una capacidad bélica, en muchas ocasiones mayor a la del Estado, que garantiza el cumplimiento de las reglas sociales y de uso y organización del territorio impuestas por ellas.

${ }^{2}$ Las fronteras de la legalidad se modifican con la historia y las prácticas comunitarias. A este respecto podemos constatar el deslizamiento de los límites de permisibilidad del consumo de drogas con la legalización de la marihuana, que implica cambios legales y de prácticas sociales, y una revisión conceptual para justificar el trato diferenciado de los diversos tipos de estupefacientes que circulan en el mercado y el cambio en el tratamiento de la marihuana. 
Estudiosos del tema como Hansen y Stepputat (2006) caracterizan el fenómeno claramente referido al concepto de soberanía y denominan estas otras espacialidades como soberanías de facto, concepto retomado por David Barrios Rodríguez para trabajar este proceso de disputa por el poder y la soberanía en lugares como México, Colombia o Río de Janeiro donde estos grupos “... prefiguran la instauración de órdenes sociales paralelos a través de los cuales el capitalismo contemporáneo encuentra formas de reproducción que traspasan las fronteras constituidas de manera previa y que se esparcen en diversos ámbitos" (Barrios Rodríguez, 2021, p. 4), estableciendo "órdenes de autoridad paralelos al de los Estados, en el marco de los cuales imponen regulaciones sobre las formas de convivencia, comportamiento, e inclusive sancionan conductas a través de penas y castigos" (Barrios Rodríguez, 2020, p. 13).

Al observar en un mapa las regiones tomadas o controladas por los diferentes cárteles en un país como México, resulta evidente ese traslapamiento de soberanías. ¿Qué es el Estado en estas circunstancias? ¿Cuál es su margen de acción? ¿En qué se soporta la soberanía del Estado si no es en estas otras soberanías que le carcomieron los cimientos?

\section{Sociedad/conflictos sociales}

El cuarto jinete del Apocalipsis, en referencia al Estado-nación, está implícito en la trampa de la constitución de Estados unitarios, supuestamente huéspedes de una comunidad consensuada.

Pablo González Casanova acuñó el concepto de colonialismo interno para referir las relaciones de subordinación y conflicto entre los diversos grupos sociales que componen lo que se suele llamar comunidad nacional, caracterizada por "contradicciones entre el gobierno nacional y las nacionalidades neocolonizadas" (González Casanova, 2003, p. 2), destacando no solo las diferencias de nivel entre ellos, sino, sobre todo, las que atañen a sus modos de vida, de organización social y de ejercicio de la política. Es decir, considerando que la formación de los Estados cayó por encima de la miscelánea de societalidades, cosmovisiones y modos de vida que los preexistían, como mecanismo de disciplinamiento general, cultural, civilizatorio. Con la formación de los Estados-nación se promovió una visión societal única y excluyente.

En la región americana, que experimentó una invasión en gran escala, acompañada de políticas de negación y vaciamiento cultural, el proceso de homogeneización combinó medidas de corte militar, como el agrupamiento de los pueblos originarios en aldeas en vez de territorios extendidos y dispersos, o como la imposición de figuras caciquiles para focalizar la interlocución y el control, con medidas evangelizadoras de vaciamiento 
e imposición de sentidos y negación de la memoria histórica de larga duración que tenían esos pueblos. Las quemas de libros, la construcción de nuevas edificaciones, diseñadas de acuerdo a claves culturales de los invasores, sobre las de los pueblos o naciones que habitaban las tierras americanas o del Abya-Yala antes de la invasión, la negación de las lenguas y costumbres, además de las masacres, saqueos y la guerra biológica, fueron la ruta de doblegamiento y explotación trazada por los invasores, aun antes de que el capitalismo en desarrollo prefigurara la organización territorial mediante el afianzamiento de Estados-nación.

Las poblaciones americanas fueron reducidas a una décima parte de lo que eran antes de la llegada de los invasores y una parte de ellas fueron esclavizadas o subordinadas. No obstante, se rebelaron, resistieron y hasta hoy mantienen la reivindicación de sus visiones del mundo y formas de vida, como lo testimonia la ola de levantamientos continentales con motivo de los 500 años.

Ya con la conformación de los Estados, resultado de las insurrecciones independentistas, se impusieron fronteras que cortaban los territorios ancestrales para repartirlos entre Estados vecinos y se instituyó la propiedad privada. Los pueblos originarios, a pesar de haber contribuido con la lucha por la expulsión de los invasores, fueron, en la mayoría de los casos, despojados de sus tierras o replegados hacia territorios agrestes o alejados de los elegidos para el asentamiento de las buenas sociedades a las que ellos no pertenecían. Ajenos a las lógicas individualistas y a la propiedad privada y convencidos de sus prácticas comunitarias, estos pueblos se mantuvieron en alerta o en resistencia frente a los avatares de la nueva fase colonizadora.

En un afán por lograr la unidad nacional, tan necesaria para el fortalecimiento y cohesión del Estado, se diseñaron políticas integracionistas muy agresivas que, como nuevos hechos evangelizadores, trataban de educar a estos pueblos imbuyéndolos de la visión moderna del mundo a cambio del abandono de la suya propia. La diversidad simplemente no se aceptaba, y mucho menos la visión y los modos de los no-occidentalizados, entre los que se cuentan también las comunidades formadas por las poblaciones de origen africano que llegaron a América en calidad de esclavas, que si no eran integradas eran combatidas por todos los medios.

No obstante, a pesar de la fuerte embestida desestructuradora/ asimiladora que significó la descolonización política y la constitución de los Estados independientes, las diversidades persistieron y más de cinco siglos después siguen en conflicto, peleando su espacio y la pervivencia de sus modalidades organizativas para la reproducción de la vida. El problema no está resuelto. La soberanía del Estado-nación no es equivalente a la soberanía del pueblo, es más bien su negación. 
Pero si esto ocurre con respecto a los sistemas de vida en confrontación o en disputa no solo por el espacio, sino por el sentido de realidad, como pueden ser los pueblos indígenas o las comunidades de origen africano, un quiebre adicional o entrecruzado se presenta a partir de las definiciones conceptuales de lo nacional desde el punto de vista de las clases y de la autodeterminación colectiva en un colectivo fuertemente dispar y polarizado. La nación, lejos de ser homogénea, se conforma de naciones o grupos organizativa y culturalmente diversos que solo circunstancialmente encuentran espacios de confluencia e identidad compartida. El Estado representa la frontera de su imposibilidad y, por tanto, ni lo reconocen ni se reconocen en él.

\section{Territorio y Estado}

Las comunidades, los pueblos, las societalidades colonizadas o incluso negadas en su especificidad coinciden con la comunidad nacional ilusoria en la defensa del territorio. El problema es que para salvaguardar sus territorios y territorialidades (modos de vida y organización integral) requieren enfrentarse justamente al Estado-nación. Ante una invasión extranjera flagrante, evidentemente la coincidencia es inmediata, pero no así ante las otras formas de invasión externa como las inversiones mineras o extractivas en general, las del agronegocio, la construcción de megainfraestructuras (aun cuando estén a cargo del Estado nacional), explotaciones forestales u otras que tienen una implicación territorial directa. Estas intervenciones son celebradas por el Estado mientras que los grupos poblacionales afectados las repudian y tratan de impedirlas. No es solo un asunto de legitimidad, sino de disputa de visiones y de territorios.

Los territorios tienen una historia de construcción mucho más larga que los Estados. Los Estados se trazaron sobre territorios forjados, que encierran memorias y tradiciones milenarias cuyas raíces profundas no hacen fácil su erradicación. La solidez de los entramados territoriales, ecocosmológicos, resalta notablemente ante la de Estados que no terminan de fraguar todo lo que llevan dentro. Mucho más en referencia a América Latina y el Caribe donde su fragilidad frente al supraestado norteamericano los pone en condiciones de subalternidad cuestionando su autoridad soberana.

Sin demeritar ni un ápice la relevancia y la eficacia política de las luchas antiimperialistas, es insoslayable reflexionar, sin complacencias, sobre el Estado como forma de disciplinamiento jerárquico y su relación con la real comunidad de comunidades que supuestamente lo integran; sobre su pertinencia ante poderes que lo rebasan, le imponen reglas y políticas y lo mantienen solo como fachada; y sobre su historicidad, que está siendo reventada por dentro y por fuera. 
Las instituciones responden a las circunstancias de su tiempo. Tienen límites y fronteras. Las fuerzas que las crean están vivas, se transforman con el tiempo. Los Estados-nación generados por un capitalismo emergente y en consolidación son traspasados, destruidos y violentados por el capitalismo maduro y en proceso de decadencia civilizatoria. ¿Dónde están en este proceso y circunstancia los pueblos que luchan por autodeterminación y por el reconocimiento de sus diversidades? ¿Cómo se colocan frente a los agentes de la destrucción: corporaciones, cárteles y Estados? ¿Cómo vislumbramos las disyuntivas sistémicas desde nuestros lugares de enunciación, latinoamericanos y caribeños, y nuestra larga historia sobre y con el planeta? ¿Se puede pensar en organizaciones colectivas no capitalistas? ¿Será que el Estado logra navegar esta deriva?

\section{Referencias}

Banco Mundial (2019). PIB Mundial. https://data.worldbank.org Barrios Rodríguez, David (2020). La vida entre cercos: militarización social en América Latina en el Siglo XXI [Tesis de doctorado]. PPELA, UNAM, México. http://ru.atheneadigital.filos.unam.mx/jspui/ handle/FFYL_UNAM/490

Barrios Rodríguez, David (2021). Tesis sobre la militarización social en América Latina y el Caribe. Observatorio Latinoamericano de Geopolítica. https://geopolitica.iiec.unam. $\mathrm{mx} /$ sites/geopolitica.iiec.unam.mx/files/2021-10/Tesis\%20 sobre\%20la\%20militarizaci\%C3\%B3n\%20social\%20en\%20 Am\%C3\%A9rica\%20Latina\%20y\%20el\%20Caribe_David\%20 Barrios_Rodr\%C3\%ADguez\%20\%281\%29.pdf

Ceceña, Ana Esther (2014). La dominación de espectro completo sobre América. Patria (Ministerio de Relaciones Exteriores, Comercio e Integración del Ecuador), (1), 85-103.

Ceceña, Ana Esther (2017). Chevron: La territorialidad capitalista al límite. Chevron. Paradigma de la catástrofe civilizatoria. México: Siglo XXI.

Fortune (2019). Ranking. Fortune Global 500-2019. https://www.fortuneenespanol.com/rankings/ranking-fortune-global-500-2019/ amp/

González Casanova, Pablo (2003). Colonialismo interno: una redefinición. Conceptos y fenómenos fundamentales de nuestro tiempo. México: UNAM.

Hansen, Thomas Bloom y Stepputat, Finn (2006). Sovereignty revisited. Annual Review of Anthropology, 35, 295-315. 